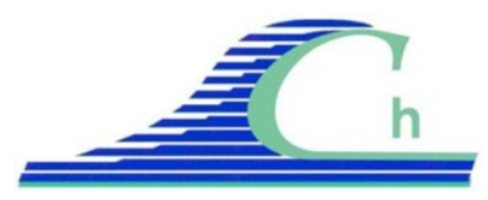

XII ${ }^{\text {ìmes }}$ Journées Nationales Génie Côtier - Génie Civil

Cherbourg, 12-14 juin 2012

DOI:10.5150/jngcgc.2012.102-H（C) Editions Paralia CFL

disponible en ligne - http://www.paralia.fr - available online

\title{
Caractérisation du bilan de circulation hydrologique du lagon de Wallis (Pacifique sud-ouest).
}

\author{
Thierry HOIBIAN ${ }^{1}$, Michel ALLENBACH ${ }^{1}$
}

1. PPME-EA 3325. Université de la Nouvelle-Calédonie, BP R4, 98851 Nouméa Cedex, Nouvelle Calédonie.

hoibian@univ-nc.nc,allenbach@univ-nc.nc

\section{Résumé :}

Une étude océanologique du lagon de Wallis est en cours de réalisation sur financements de l'IFRECOR (Initiative Française pour les Récifs Coralliens). Elle s'inscrit dans un programme de recherche pluri-annuel mené sur le Territoire des Îles Wallis et Futuna. Ce programme vise à caractériser l'environnement météo-océanologique du lagon et la dynamique sédimentaire littorale pour lutter contre les tendances érosives constatées. Il vise à plus long terme, à l'estimation des effets du changement climatique sur le littoral, ainsi qu'à la définition des stratégies d'adaptation à développer dans le cadre de la GIZC (Gestion Intégrée des Zones Côtières). L’une des problématiques scientifiques en aval du programme appliqué est la compréhension de la courantologie des lagons coralliens en mers mésotidales. A titre d'exemple, nous présentons le bilan de circulation des courants de marée au travers des passes du lagon de Wallis.

Mots clefs : Courantologie - ADCP - Lagon - Passes - Bilan de circulation - GIZC Pacifique sud ouest - Territoires insulaires - Wallis - Réchauffement climatique

\section{Abstract:}

An oceanologic study of Wallis islands lagoon is currently in progress on funding IFRECOR (French Initiative for Coral Reefs). 2008). It is part of a research program conducted multi-year in the Territory of Wallis and Futuna Islands. This program aims to characterize the weather-oceanology environment of Wallis lagoon and sediment dynamics in the fight against coastal erosion trends observed. It also aims to estimate the long term impact of climate changes on the shore and the definition of adaptation strategies to setup the ICZM (Integrated Coastal Zone Management). One of the scientific issues downstream of the applied program is understanding the current patterns of coral lagoons in 'mesotidales' seas. For example, we present the results of tidal current flow through the passes of the Wallis lagoon.

Key words: Tidal currents - ADCP - lagoon - channel entrance - tidal budget - South West Pacific - Island territories - Wallis - Global warming

\section{Introduction}

Une étude océanologique est en cours de réalisation sur financements de l'IFRECOR (Initiative Française pour les Récifs Coralliens) dans le cadre d'un programme pluri- 


\section{Thème 7 - Océanographie opérationnelle et situations extrêmes}

annuel consacré à la caractérisation de l'environnement météo-océanologique et de la dynamique sédimentaire du lagon de Wallis. Les finalités appliquées visent à la définition des stratégies d'adaptation à développer dans le cadre de la GIZC (gestion intégrée des zones côtières) du territoire et à la pose de défenses de génie civil côtier optimisées pour lutter contre les mécanismes érosifs constatés à la côte (ALLENBACH et al., 2008). L'une des problématiques scientifiques fondamentales traitées par le programme est un apport à la compréhension de la courantologie des lagons coralliens en mers mésotidales et à la modélisation des flux respectifs passant par les passes et par-dessus les récifs-barrières. A titre d'exemple, nous présentons dans cette communication, le calcul du bilan de circulation des courants de marée au travers des passes du lagon de Wallis.

\section{Localisation}

Positionnée par $13^{\circ} 16^{\prime} \mathrm{S}$ et $176^{\circ} 15^{\prime} \mathrm{W}$, l'archipel des îles Wallis est un territoire ultramarin français situé dans le Pacifique sud-ouest. L'île principale, est longue de 15 $\mathrm{km}$ et de large de $6 \mathrm{~km}$. Elle est entourée un récif barrière qui s'étire sur $24 \mathrm{~km}$ dans la direction nord-sud et sur $15 \mathrm{~km}$ dans une direction est-ouest. Le récif délimite un lagon d'une largeur de 3 à $5 \mathrm{~km}$, d'une profondeur moyenne d'environ $20 \mathrm{~m}$ avec des fosses où elle dépasse $50 \mathrm{~m}$. Ce lagon est ouvert sur l'océan au niveau de quatre passes, une au sud (Honikulu) et trois dans la partie ouest de l'île (Avatolu, Fuga Uvea et Fatumanini) (figure 1).

\section{Matériel et méthodes}

Une importante base de données océanologique a été constituée à partir des enregistrements de différents instruments :

- un houlographe-marégraphe SBE 26 de Seabird Electronic implanté sur une station de référence de la rade de Mata Utu (figure 1) pour la mesure du niveau marin, des vagues, des marées et de la température de l'eau.

- un profileur de courant Nortek $600 \mathrm{kHz}$ qui enregistre les vitesses et directions des courants sur l'ensemble de la tranche d'eau et qui a été implanté sur de nombreux sites du lagon et des passes (figure 1). Cet instrument permet aussi l'enregistrement des houles directionnelles, de la température de l'eau et de la pression hydrostatique.

La durée d'une session qui est déterminée par l'encombrement mémoire du SBE26, s'étend sur 74 jours environ en moyenne.

La base de données environnementale constituée à partir de nos propres enregistrements a été complétée par l'intégration des données fournies par de nombreux organismes nationaux et internationaux; Météo France Nouvelle-Calédonie pour les enregistrements de la station de Hihifo (Aéroport de Wallis), Le SHOM pour les prévisions de marée du port de Mata Utu, ainsi que par les données des serveurs d’océanographie numérique des programmes Mercator-Océan (http://www.mercator- 


\section{XII ${ }^{\text {èmes }}$ Journées Nationales Génie Côtier - Génie Civil \\ Cherbourg, 12-14 juin 2012}

ocean.fr), Aviso-Oceanobs (http://www.aviso.oceanobs.com/fr/) et Iowaga pour ce qui est des conditions océanologiques et météorologiques générales du Pacifique sud ouest.

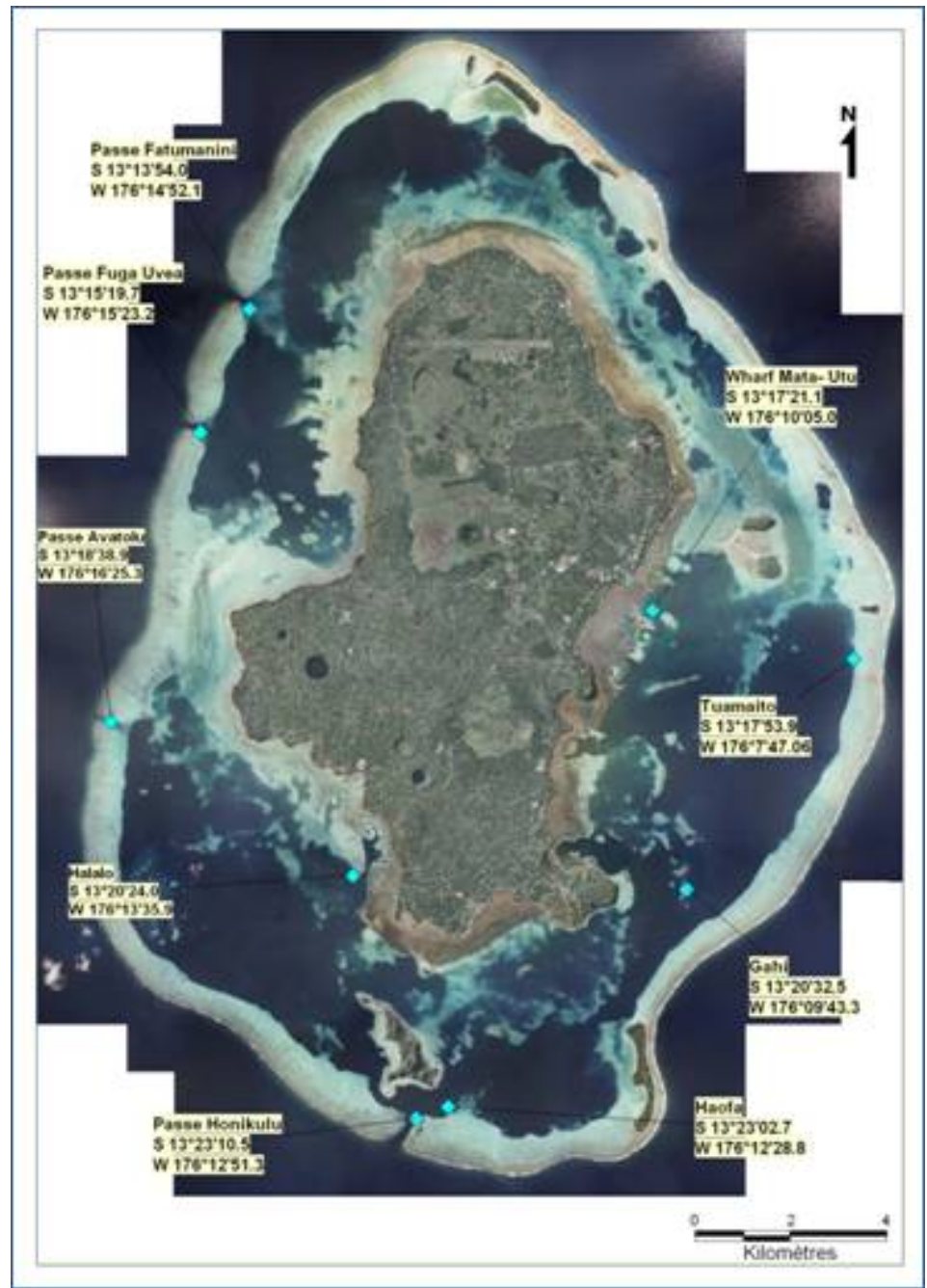

Figure 1. Localisation des stations du lagon de Wallis.

Les données et les résultats des analyses de cette étude sont mis à disposition par le biais d'un serveur cartographique hébergé par l’Université de la Nouvelle-Calédonie, à l'adresse web http://windvane.univ-nc.nc/ ifrecor/.

\section{Traitements des données de courantologie}

Le traitement des données et le tracé des résultats ont été effectués principalement à partir des logiciels des constructeurs d'instruments et de routines Matlab. Deux approches sont utilisées, une analyse statistique globale pour l'ensemble des données d'une session, indépendamment du temps et une analyse statistique détaillée par type de marée. 


\subsection{L'analyse statistique globale}

Cette analyse concerne l'ensemble des données de la session par cellule. Elle donne une idée des gammes de vitesses et des regroupements de directions (figure 2) et permet de constituer un tableau des maximums et moyennes (figure 3).

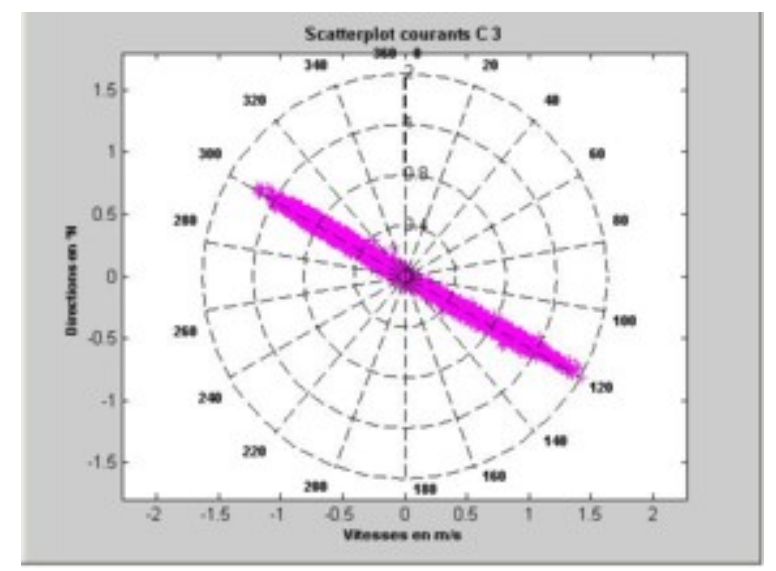

Figure 2. Diagramme de dispersion des vecteurs vitesse pour la cellule C3 et l'ensemble de la session de la passe d'Avatolu.

\begin{tabular}{|c|c|c|c|c|c|c|c|c|c|}
\hline Passes & \multicolumn{3}{|c|}{ Avatolu } & \multicolumn{3}{|c|}{ Honikulu } & \multicolumn{3}{|c|}{ Fuga Uvea } \\
\hline Cellule & $\begin{array}{c}\text { Prof } \\
(\mathrm{m})\end{array}$ & $\begin{array}{c}\begin{array}{c}\text { Vmoy } \\
(\mathrm{m} / \mathrm{s})\end{array} \\
\end{array}$ & $\begin{array}{c}\operatorname{Vmax} \\
(\mathrm{m} / \mathrm{s})\end{array}$ & $\begin{array}{l}\text { Prof } \\
\text { (m) }\end{array}$ & $\begin{array}{l}\begin{array}{l}\text { Vmoy } \\
(\mathrm{m} / \mathrm{s})\end{array} \\
\end{array}$ & $\begin{array}{l}\operatorname{Vmax} \\
(\mathrm{m} / \mathrm{s})\end{array}$ & $\begin{array}{c}\text { Prof } \\
(\mathrm{m})\end{array}$ & $\begin{array}{l}\begin{array}{l}\text { Vmoy } \\
(\mathrm{m} / \mathrm{s})\end{array} \\
\end{array}$ & $\begin{array}{l}\operatorname{Vmax} \\
(\mathrm{m} / \mathrm{s})\end{array}$ \\
\hline$C 1$ & $-2,5$ & 0,72 & 1,60 & $-2,30$ & 0,73 & 1,72 & $-1,0$ & 0,45 & 1,03 \\
\hline$C 2$ & $-4,0$ & 0,56 & 1,55 & $-4,30$ & 0,55 & 1,56 & $-2,5$ & 0,67 & 1,57 \\
\hline C3 & $-5,5$ & 0,63 & 1,63 & $-6,30$ & 0,61 & 1,62 & $-4,0$ & 0,40 & 1,05 \\
\hline$C 4$ & $-7,0$ & 0,58 & 1,56 & $-8,30$ & 0,60 & 1,61 & $-5,5$ & 0,40 & 1,10 \\
\hline C5 & $-8,5$ & 0,49 & 1,40 & $-10,30$ & 0,57 & 1,53 & $-7,0$ & 0,38 & 1,01 \\
\hline C6 & - & - & - & $-12,30$ & 0,53 & 1,37 & $-9,5$ & 0,34 & 0,93 \\
\hline
\end{tabular}

Figure 3. Statistique globale des vitesses de courant par cellule pour les trois passes.

\subsection{L’analyse statistique détaillée}

Elle est organisée en fonction des différents cycles de marée à 6 heures et à 15 jours. Le cycle de base à 6 heures est décomposé en heures marées (HM) dont la durée varie d'une marée sur l'autre. Ce découpage permet d'établir une statistique des vitesses et directions des courants qui n'est valable que sur une période assez courte de quelques jours (3 à 5) et de caractériser les vitesses et directions, maximales et moyennes, des courants pour chaque heure marée type de $\mathrm{PM}+1, \mathrm{PM}+2, \ldots$ à $\mathrm{PM}+12$, (figure 4). 


\begin{tabular}{|c|c|c|c|c|c|c|c|c|c|c|c|c|c|}
\hline & PM & +1 & +2 & +3 & +4 & +5 & +6 & +7 & +8 & +9 & +10 & +11 & +12 \\
\hline \multirow{2}{*}{ VE } & Vit moy (m/s) & 0,53 & 0,77 & 0,96 & 1,22 & 1,00 & 0,13 & 1,08 & 1,30 & 1,13 & 0,36 & 0,05 & 0,31 \\
\hline & Dir Vmoy $\left({ }^{\circ} N\right)$ & 293 & 291 & 296 & 297 & 299 & 196 & 121 & 121 & 122 & 126 & 268 & 283 \\
\hline \multirow{2}{*}{ ME } & Vit moy (m/s) & $\mathbf{0 , 1 0}$ & 0,05 & 0,45 & 0,74 & 0,87 & 1,04 & 1,11 & 1,14 & 1,20 & 1,14 & 0,95 & 0,65 \\
\hline & Dir Vmoy $\left({ }^{\circ} N\right)$ & 309 & 337 & 306 & 305 & 304 & 306 & 304 & 306 & 304 & 303 & 305 & 312 \\
\hline
\end{tabular}

Figure 4: Exemple de tableau résultant de l'analyse courantologique détaillée.

\section{Résultats}

Les observations réalisées sur les trois passes, Honikulu, Fuga Uvea et Avatolu, montent de grandes similitudes dans les régimes hydrologiques de ces sites et permettent d'établir un schéma de circulation par type de marée (figure 5) :

- en vives eaux, le courant circule dans les passes suivant un modèle classique avec un courant rentrant pendant le flot et un courant sortant pendant le jusant. Les vitesses sont d'autant plus élevées que l'amplitude de marée est plus forte.

- en mortes eaux, il se produit une vidange continuelle du lagon vers l'océan. Les vitesses des courants ralentissent pendant le flot et vont jusqu'à s'annuler à PM+2. Ce processus se poursuit pendant plusieurs jours successifs (voir figure 8).

\begin{tabular}{|l|c|c|c|c|}
\hline Mata Utu & PM VE & PM $\mathbf{M E}$ & BM VE & BM $\mathbf{M E}$ \\
\hline Hauteur (m) & 1,80 & 1,5 & 0,5 & 0,8 \\
\hline Amplitude (m) & 1,30 & 0,70 & \multicolumn{2}{|c|}{ Semi diurne } \\
\hline
\end{tabular}

Figure 5. Hauteur des marées rapportées au zéro hydrographique à Mata Utu.

\section{Bilan de circulation}

Dans le but de quantifier le phénomène, il a été effectué un calcul du bilan de circulation pour la durée totale d'un cycle de marée, selon la formule développée cidessous. Les vecteurs courant sont projetés sur l'axe de la passe par produit avec le cosinus de la différence angulaire. Un signe positif est assigné au courant de flot et un signe négatif au courant de jusant.

$$
V o l=S \int_{t 0}^{t i} V(t) \times \cos (\theta-\alpha(t)) d t
$$

où :

Vol = volume d'eau ayant transité entre l'instant $t_{0}$ et l'instant $t_{i} \quad\left(\right.$ en $\left.^{3}{ }^{3}\right)$.

$S$ = surface unité $\left(\right.$ en $\left.^{2}{ }^{2}\right)$.

$V=$ vitesse du courant (en $\mathrm{m} / \mathrm{s}$ ).

$t=$ temps écoulé depuis l'instant $t_{0}$ (en s).

$\theta=$ axe de la passe par rapport au nord $\left(^{\circ}\right)$.

$\alpha=$ direction du vecteur courant par rapport au nord $\left(^{\circ}\right)$.

$\mathrm{dt}=$ différentielle du temps. 


\subsection{Bilan de circulation sur un cycle de marée}

A titre d'exemple, nous considérons un cycle de marée de vives eaux, la session de la passe d'Avatolu et la cellule C3 située sensiblement à mi-profondeur (figure 5). Ces graphiques des bilans de circulation permettent de constater que les écoulements résultants sont toujours dirigés vers l'océan, indiquant une vidange du lagon au niveau des passes quelque soit le type de marée (figure 6).

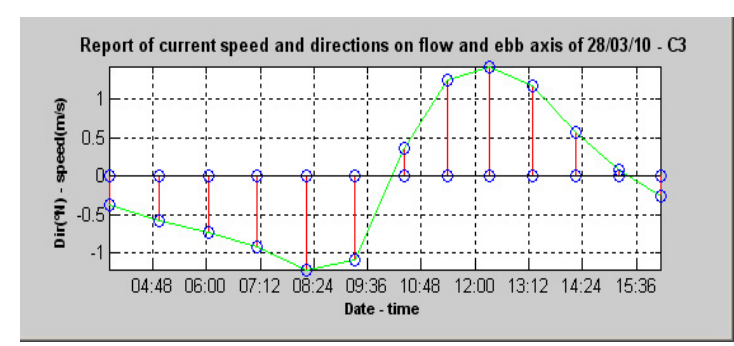

En vives eaux

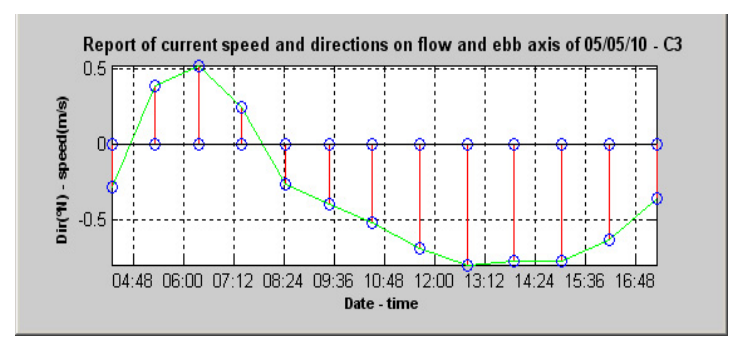

En mortes eaux

Figure 6. Bilans de circulation à la Passe d'Avatolu pour la cellule C3.

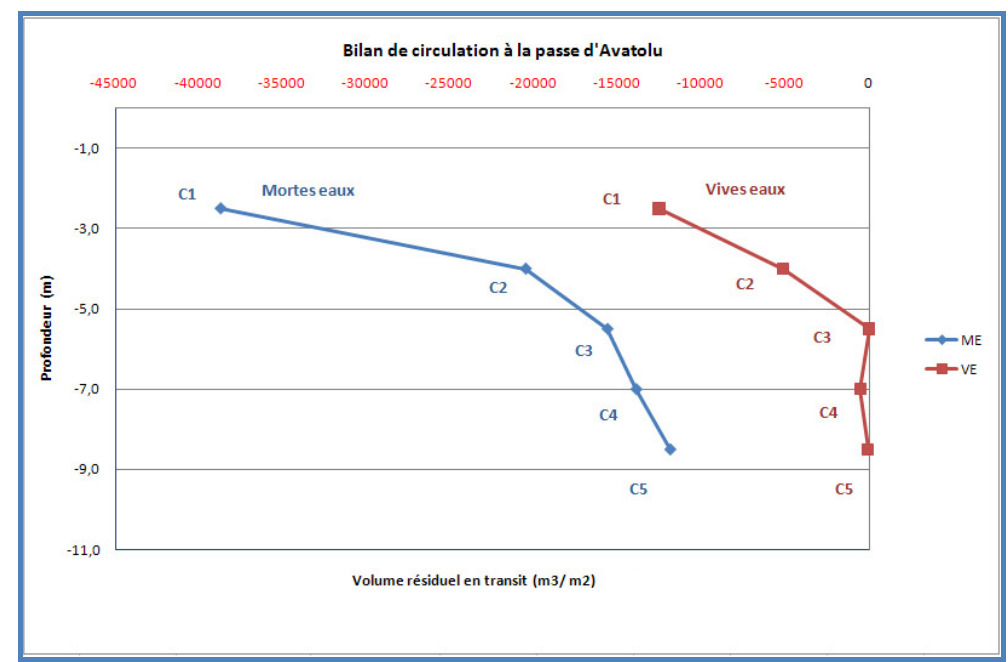

Figure 7. Comparaison des bilans de circulation sur un cycle de marée en vives eaux et en mortes eaux, à la passe d'Avatolu.

\subsection{Bilan de circulation général}

Après le calcul du bilan résiduel sur un cycle de marée, la méthode est appliquée sur l'ensemble d'une session afin d'examiner le continuum des échanges entre le lagon et l’océan (figure 8).

Ces graphes tracés pour les trois passes principales, montrent tous, un écoulement résiduel dirigé majoritairement vers le jusant et indiquent un bilan largement déficitaire pour toutes les sessions. Il arrive assez exceptionnellement, pendant les fortes marées de vives eaux que les bilans s'équilibrent entre le flot et le jusant ce qui se traduit par la présence d'un palier sur la courbe (figure 9). 


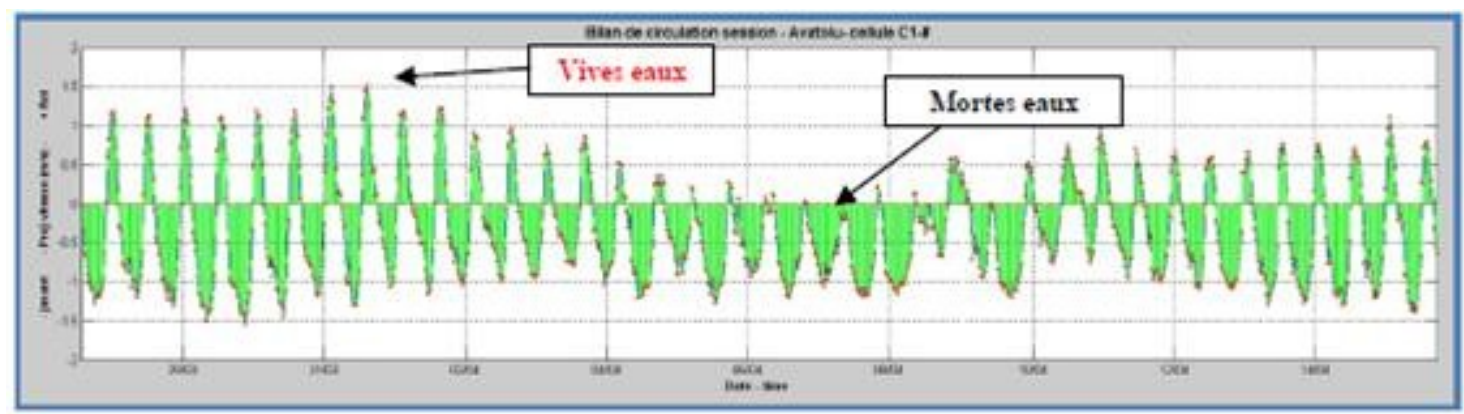

Figure 8. Graphe des vitesses projetées pendant une partie de la session.

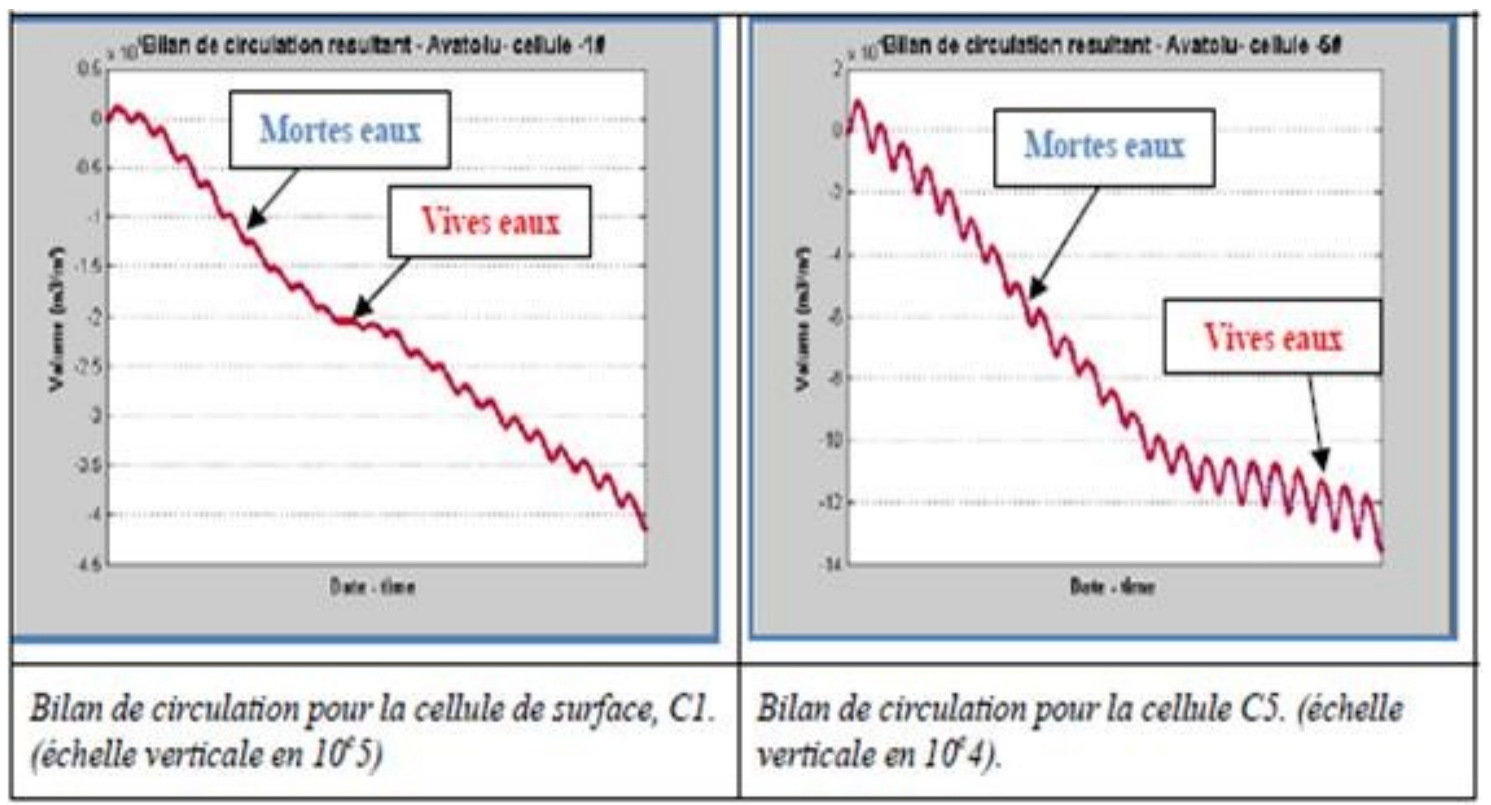

Figure 9. Bilans de circulation résultant de la passe d'Avatolu.

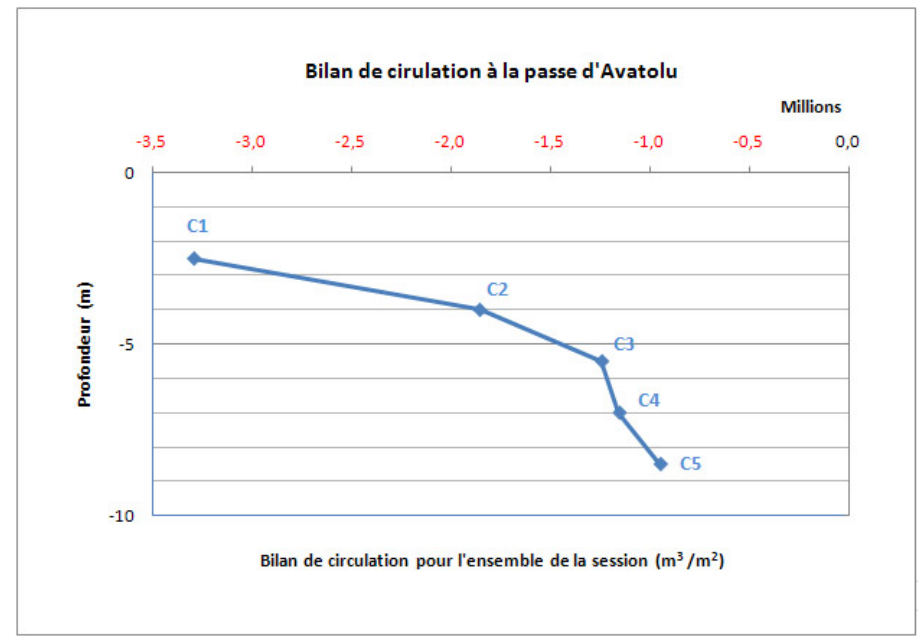

Figure 10. Graphe du bilan de circulation de chaque cellule, pour l'ensemble de la session à la passe d'Avatolu. 
Ces bilans montrent que la circulation par les passes consiste en un processus de vidange quasi-permanent (figure 10).

\section{Conclusions}

Ces études menées depuis 2006 dans le lagon de Wallis conduisent à une meilleure compréhension des spécificités hydrologiques du lagon et constituent aujourd'hui les éléments d'une base de données de référence pour le suivi de l'impact du changement climatique dans cette partie de l'océan Pacifique. L'étude des courants de marée dans les passes a confirmé l'observation des insulaires selon laquelle les courants restaient dirigés vers le large pendant plusieurs jours (voir figure 8). Ce fait supposé lié à l'amplitude des vagues océanique par RICHARD et al., 1982, apparaît finalement comme davantage en relation avec le type marée. Les bilans de circulation déficitaires au travers des passes, notamment pendant les marées de mortes eaux, mettent en évidence le fait que le remplissage du lagon par-dessus la barrière récifale joue un rôle important dans les échanges avec l'océan. La circulation par les passes consiste globalement en un processus de vidange prépondérant.

\section{Remerciements :}

Nos remerciements sont adressés aux organismes qui sont à l'origine de ces études et ont participé à leurs financements ; l'IFRECOR, le MOM, l'ONERC, Météo-France NC et le SERVICE de l'ENVIRONNEMENT de Wallis et Futuna pour son aide logistique.

\section{Références bibliographiques}

RICHARD G., BAGNIS P., BENNETT J., DENIZOT M., GALZIN R., RICARD M., SALVAT B. (1982). Étude de l'environnement lagonaire et récifal des Iles Wallis et Futuna (Polynésie occidentale). Territoire de Wallis et Futuna - Antenne MuséumE.P.H.E.

ALLENBACH M., HOIBIAN T. (2008). Enjeux, spécificités et perspectives de la gestion intégrée du littoral dans l'espace insulaire français du sud-ouest Pacifique. European Journal of Environmental and Civil Engineering, Vol. 12, $n^{\circ}$ 1-2/2008, pp 15-34, Revue européenne de génie civil. 